\title{
Evaluación del Sistema de Vigilancia de VIH - SIDA y Mortalidad por SIDA en Caquetá, Colombia durante el período 2014-2018
}

\author{
Shirley Sáenz ${ }^{\text {a }}$, Lilian Andrea Rodríguez Gutiérrez ${ }^{\text {b }}$, Gloria Suarez ${ }^{\text {c }}$, Alfonso Campo ${ }^{\text {d }}$ \\ a Magíster en Salud Pública. Instituto Nacional de Salud shirleysaenz@hotmail.com \\ ${ }^{\mathrm{b}}$ Especialista en Epidemiología General. Instituto Nacional de Salud

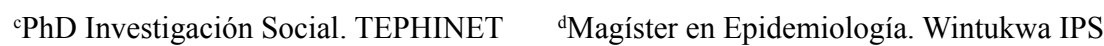

DOI 10.22517/25395203.21951

\section{Resumen}

Introducción: La pandemia por el Virus de Inmunodeficiencia Humana (VIH) y el Síndrome de Inmunodeficiencia Adquirida (SIDA) han generado numerosas pérdidas humanas, deterioro de la calidad de vida y consecuencias económicas negativas. Se han implementado programas de prevención para fortalecer el diagnóstico temprano y frenar la transición del VIH al SIDA.

Objetivo: Hacer seguimiento de la notificación de VIHSIDA para evaluar los atributos cualitativos y cuantitativos, de acuerdo con los procesos establecidos para la notificación al SIVIGILA, en Caquetá, Colombia.

Métodos: Fue utilizada metodología de evaluación de sistemas de vigilancia de los CDC. Para evaluar los atributos cualitativos se realizaron encuestas virtuales a 47 responsables del sistema en diferentes entes territoriales. Para evaluar los atributos se revisaron documentos y bases de datos. Se realizó análisis cualitativos mediante triangulación en Epi-info y análisis cuantitativos usando medidas de frecuencia.

Resultados: a)Simplicidad: es fácil el diligenciamiento de registros; b)Flexibilidad: el subsistema ha tenido diferentes cambios en definición de caso e inclusión de variables; c) Aceptabilidad: se reconoce la importancia de la vigilancia de VIH - SIDA y Mortalidad por SIDA; d) Oportunidad: No es oportuno, no se tiene una periodicidad establecida para el análisis de la información; e)Representatividad: no representan el total de los casos del territorio; f)Utilidad: el subsistema permite formulación de políticas públicas; g)
Calidad: hubo 20 casos repetidos; h) Sensibilidad: no capta todas las mortalidades.

Palabras clave: Evaluación, sistema de vigilancia epidemiológica, VIH-SIDA.

Evaluation of the HIV - AIDS and the AIDS Mortality Surveillance System in Caquetá, Colombia, between 2014 and 2018.

\begin{abstract}
Introduction: HIV/AIDS is a global pandemic that has generated numerous human losses, life's quality deterioration, and unfavorable economic consequences. Several prevention programs have been implemented to strengthen early diagnosis and stop the transition from HIV to AIDS.

Objective: To evaluate and follow-up on the qualitative and quantitative attributes of the HIV-AIDS notification process according to the established procedure of notification to the SIVIGILA in Caqueta, Colombia.

Methods: Centers for Disease Control and Prevention's methodology was used for evaluating the subsystem. Virtual surveys were carried out in 47 people in charge of the system in different territorial entities, and documents and databases were reviewed to examine qualitative attributes. In terms of analysis, triangulation in Epi-info was made in qualitative aspects, and frequency measurements were used in quantitative aspects.
\end{abstract}

Results: The results are divided into eight categories. a)Simplicity: it is easy to complete the records. b)Flexibility: the subsystem has had different changes in terms of case definition and the inclusion of variables. c)Acceptability: it is recognized the importance of monitoring HIV - AIDS and AIDS Mortality. d)Opportunity: It is not opportune; there is no periodicity for the information analysis. e)Representativeness: the total number of cases in the territory is not represented. f)Utility: the subsystem allows the formulation of public policies. g)Quality: there were 20 repeated cases. h)Sensibility: all mortalities are not taken into account.

Keywords: Evaluation, epidemiological surveillance system, HIV-AIDS. 


\section{Introducción}

La pandemia por el Virus de Inmunodeficiencia Humana (VIH) y el Síndrome de Inmunodeficiencia Adquirida (SIDA) representa uno de los mayores desafíos para la humanidad ya que ha generado numerosas pérdidas humanas, deterioro de la calidad de vida de millones de personas, consecuencias económicas negativas derivadas del incremento de los recursos necesarios para la atención en salud y de la incapacidad y muerte de miles de personas en edad productiva. Al final de 2001, se calculaban 40 millones de personas viviendo con VIH - SIDA, de los cuales 37,1 millones son adultos y 3 millones, menores de 15 años; las mujeres constituyen el 49,8\% de los adultos infectados. Las nuevas infecciones de 2015 alcanzaron 36,7 millones de personas infectadas; en este mismo año se registraron 2,1 millones de personas que contrajeron la enfermedad, entre ellas 150.000 niños, y 1,1 millones murieron a causas relacionadas al SIDA $_{(1)}$.

La infección por el VIH-SIDA ha sido considerada en el sistema general de seguridad social en salud como una patología de alto costo, por generar un fuerte impacto económico sobre las finanzas del sistema general de seguridad social en salud y por causar un dramático efecto sobre la calidad de vida de los pacientes y su familia, incluidas las repercusiones laborales. Se estima que el manejo del VIH-SIDA ha comprometido en los últimos años el $0,5 \%$ del gasto en salud del país y cerca del $1 \%$ del gasto en seguridad social en salud. Frente a esta situación, y no sólo sobre la base de las implicaciones económicas, sino frente a la calidad de vida y de la atención en los servicios de salud, en diferentes ámbitos se ha venido planteando la necesidad de implementar programas de prevención primaria y secundaria, fortalecer el diagnóstico temprano, definir y aplicar protocolos de tratamiento, frenar la transición del VIH al SIDA y disminuir los costos de hospitalización y complicaciones, la incapacidad y la mortalidad ${ }_{(1)}$.

Las infecciones de transmisión sexual (ITS) son un problema de salud pública, por lo que es necesario conocer su comportamiento para poder realizar acciones de promoción y prevención que permitan su control y por ende lleven a que estas se disminuyan $_{(2)}$.

Dentro de las infecciones de transmisión sexual se encuentra el VIH, donde su forma tardía es el SIDA, el cual también puede transmitirse de la madre al hijo durante el embarazo, el parto y la lactancia e igualmente por transfusiones sanguíneas, o en usuarios de drogas intravenosas ${ }_{(2)}$.

Desde que el SIDA se identificó por primera vez a comienzos de la década de los ochenta, un número sin precedentes de personas se vieron afectadas por la epidemia mundial de SIDA. Actualmente, se calcula que hay 36,7 millones de personas que viven con VIH-SIDA. África subsahariana enfrenta la carga más grande del VIH-SIDA a nivel mundial, representa el 65 $\%$ de todas las nuevas infecciones por el VIH. Otras regiones afectadas de manera significativa por el VIH-SIDA son Asia y el Pacífico, Latinoamérica y el Caribe, Europa Oriental y Asia Central $_{(3)}$.

El número total de personas que viven con el VIH ha aumentado como consecuencia del número continuado de nuevas infecciones contraídas cada año. África subsahariana sigue siendo la región más afectada por el VIH, ya que le corresponden el $65 \%$. Por raza y origen étnico, los afroamericanos son los más afectados. En el 2015, los afroamericanos conformaban solo el $12 \%$ de la población estadounidense, pero tenían el $45 \%$ de todos los nuevos diagnósticos de VIH, seguidos de los hispanos o latinos.

En Colombia, el primer caso de SIDA se notificó en 1983. Cinco años después, en 1988, se realizó el primer estudio de sero prevalencia con el propósito de intentar conocer la magnitud de la epidemia y responder a ella. A partir de entonces, el Ministerio de Salud y Protección social ha puesto en marcha, de diferentes formas, programas de vigilancia epidemiológica, principalmente entre los grupos identificados como de mayor vulnerabilidad ${ }_{(3)}$.

Colombia tiene una epidemia concentrada, es decir, que la enfermedad se presenta principalmente en grupos de alta vulnerabilidad: trabajadores y trabajadoras del sexo, hombres que tienen sexo con hombres (HSH), con una prevalencia que está por encima del $5 \%$ en el grupo de HSH, mientras que en el resto de población es menor del $1 \%{ }_{(3)}$.

Paralograrelobjetivodelpresenteestudio, serealizóseguimiento de la notificación de casos de VIH-SIDA en Caquetá, Colombia, para evaluar los atributos cualitativos y cuantitativos, de acuerdo con los procesos establecidos para la notificación al SIVIGILA, a fin de generar información oportuna, válida y confiable para orientar medidas de prevención y control.

\section{Materiales y métodos}

Se realizó la evaluación del sistema de vigilancia epidemiológica del VIH-SIDA y Mortalidad por SIDA en Caquetá utilizando la metodología propuesta por el Centro para el Control de Enfermedades CDC de Atlanta que propone seis pasos para evaluar los sistemas de vigilancia en salud pública ${ }_{(4)}$.

La población del estudio son las entidades municipales del departamento de Caquetá, los registros de los casos de VIH-SIDA y Mortalidad por SIDA en Caquetá, notificados al sistema de vigilancia en salud pública -SIVIGILA - entre 2014 y 2018 y los registros de los casos de fallecimientos en el departamento por SIDA, registrados en el certificado de 
defunción y sistematizados por el Departamento Nacional de Estadísticas de Colombia -DANE- entre 2014 y 2018.

En la base de datos se tuvieron en cuenta las siguientes variables de razón: edad del paciente, número de personas encargadas del proceso de vigilancia y número de veces al año que cuenta con personal contratado para las actividades de vigilancia de VIH-SIDA y Mortalidad por SIDA. Las variables nominales medidas por la encuesta fueron dificultad o sencillez del aplicativo para la notificación de casos de VIH - SIDA, diligenciamiento de la ficha, interpretación de las variables, procesos de ajuste en el aplicativo; uso de la información del sistema para elaboración de boletines epidemiológicos, informes de evento, canales endémicos, indicadores, utilidad de la información para otras instituciones y percepción de los cambios realizados al sistema de vigilancia de VIH - SIDA del 2014. Como variables nominales de la base de datos de SIVIGILA y RUAF se tuvieron en cuenta código del evento, semana epidemiológica, año, municipio de procedencia, sexo, entidad administradora de planes de beneficios, tipo de seguridad, estadio clínico, mecanismo de trasmisión, tipo de prueba, condición final del caso, causa básica de muerte.

Se identificaron los actores involucrados en el sistema de vigilancia en salud pública de VIH - SIDA y Mortalidad por SIDA en Caquetá. El instrumento se validó con expertos en esta vigilancia. Se aplicó una encuesta auto administrada a los referentes de nueve entidades municipales de 16 municipios y a 38 referentes de otras ciudades del país. Después de tener la información de la encuesta realizada, se verificó la calidad del dato. Para el control de los datos se verificaron los formularios y se realizó la corrección de los errores encontrados.

Se obtuvieron en la SDS de Caquetá, las bases de datos de la notificación de VIH - SIDA y Mortalidad por SIDA de Caquetá, se realizó la revisión de los registros de vigilancia de 2014 a 2018. Asimismo, los datos de fallecimiento de mortalidad por SIDA registrados en el certificado de defunción de 2014 a 2018. Se revisaron los informes de evento, boletines epidemiológicos y manuscritos publicados. La confidencialidad de los datos se aseguró mediante la anonimización de las bases de datos.

Para realizar los análisis de las bases de datos del sistema de vigilancia y de los registros de los certificados de defunción, se utilizaron las bases depuradas, se tomaron en cuenta los certificados de defunción en que la muerte tuviera certificación médica. Las bases de datos se revisaron y depuraron en Excel (®) 2010.

Las encuestas realizadas a las entidades se tabularon empleando el software Microsoft Excel ${ }^{\circledR}$ 2010, luego de realizar control de la calidad de los datos mediante la revisión manual de las encuestas. Para la utilidad, se estableció la siguiente puntuación: Poco útil (6-12), Útil (13-20), Muy útil (21-30). Se estableció una puntación para cada atributo evaluado por los referentes del evento (Tabla 1), identificando tres categorías en el funcionamiento del sistema de vigilancia: deficiente, regular y bueno.

Tabla 1. Puntaje de la evaluación de atributos del sistema de vigilancia de VIH - SIDA y Mortalidad por SIDA en Caquetá, 2019

\begin{tabular}{|l|l|l|l|}
\hline Atributo & Deficiente & Regular & Bueno \\
\hline Simplicidad & $10-20$ & $21-31$ & $32-50$ \\
\hline Flexibilidad & $4-8$ & $9-14$ & $15-20$ \\
\hline Aceptabilidad & $6-12$ & $13-20$ & $21-30$ \\
\hline Oportunidad & $3-6$ & $6-11$ & $12-15$ \\
\hline Representatividad & $2-4$ & $5-7$ & $8-10$ \\
\hline Utilidad & $5-11$ & $12-18$ & $19-25$ \\
\hline
\end{tabular}

Los datos del comportamiento del evento se organizaron en distribución de frecuencias y se presentan en tablas y gráficos. Se realizan análisis descriptivos, cálculo de proporciones, porcentajes y cálculos de medidas de tendencia central y de dispersión. Asimismo, se calculan riesgos relativos por edad, tipo de afiliación, pertenencia étnica y área de residencia con intervalos de confianza del $95 \%$.

Para evaluar la utilidad del sistema de vigilancia se verificó el cumplimiento de los objetivos del sistema, se analizaron aspectos tales como la frecuencia de los informes de evento, el contenido y la frecuencia de los boletines publicados, así como el análisis de los indicadores de VIH - SIDA y Mortalidad por SIDA en Caquetá para el período 2014 - 2018. Durante las encuestas se recolectó información sobre la utilidad de la información del sistema de vigilancia. Para el análisis de los atributos del sistema de vigilancia se realizó lo siguiente:

a. Simplicidad: Revisión de documentos, informes de eventos y encuestas para verificar el flujo de la información, integración con otros sistemas y el proceso de recolección, análisis y la divulgación de datos. Asimismo, se realizó una encuesta para verificar la comprensión de la definición de caso y los métodos de recolección de los datos.

b. Flexibilidad: Análisis de los cambios que ha tenido el sistema de VIH - SIDA y mortalidad por SIDA y su adaptación según las necesidades de información y las condiciones de operación en el tiempo.

c. Aceptabilidad: Análisis de los mecanismos de divulgación de la información. Mediante la encuesta se verificó la participación del personal de salud que opera el sistema de vigilancia de VIH - SIDA y mortalidad por SIDA y la voluntad de usar el sistema. 
d. Oportunidad: Mediante la verificación de los mecanismos para la recolección de los datos, la revisión de documentos y encuestas para verificar la oportunidad en la información generada por el sistema de vigilancia de mortalidad por SIDA y su disponibilidad para el control del evento. Igualmente, se verificaron las bases de datos y documentos mediante el cálculo del siguiente indicador: número de días desde la recolección de la información hasta la divulgación de dicha información.

e. Representatividad: Se realizó análisis de la información del sistema de vigilancia y los registros de los certificados de defunción del DANE, describiendo número de casos, tasa de prevalencia por sexo, edad y casos por entidad municipal de residencia de los casos notificados al SIVIGILA según clasificación del riesgo por año (semaforización), usando los cuartiles para el periodo $2014-2018$.

f. Estabilidad: Fue revisado el entrenamiento a los operadores del sistema de vigilancia, la recolección-gestión-análisis de los datos y la continuidad del sistema en sus fases de implementación.

g. Utilidad: Se analizó la frecuencia de los informes, las intervenciones realizadas a los casos graves identificados, el contenido y la frecuencia de los boletines publicados, así como el análisis de los indicadores. Durante las entrevistas se indagó sobre la utilidad de la información del subsistema.

h. Calidad de los datos: Se verificó la completitud y pertinencia de la información en la base de datos del sistema de vigilancia de VIH-SIDA y mortalidad por SIDA 2014-2018 en Caquetá, se realizó cálculo del porcentaje de registros duplicados, registros repetidos y los registros de datos sin diligenciar, se consideró una variable óptima aquella diligenciada más del $90 \%$ de las veces. El porcentaje de registros duplicados se obtiene verificando los registros que pertenecen al mismo paciente con variables idénticas en todos sus campos, tales como semana epidemiológica, tipo de documento, número de documento de identidad, fecha de nacimiento y fecha de consulta. Se calculó: número de registros duplicados / número del total de registros por 100.El porcentaje de registros repetidos se obtuvo verificando los registros que pertenecen al mismo paciente con las siete variables llave; se calculó: número de registros repetidos / número total de registros $* 100$. El porcentaje de respuestas en blanco es el número de respuestas que no fueron diligenciadas en la notificación, de acuerdo con las variables de la ficha de notificación, se calculó así: número de respuestas en blanco / número total de respuestas esperadas por 100.

i. Sensibilidad: Se utilizó la metodología de captura y recaptura para realizar la comparación entre los casos registrados en los certificados de defunción del DANE y los registros de VIH SIDA y Mortalidad por SIDA notificados al SIVIGILA. j. Valor predictivo positivo: Mediante la metodología de captura y recaptura se realizó el análisis de las muertes por SIDA notificadas al Sivigila y la identificación de las mortalidades que no se notificaron al SIVIGILA, según los registros de defunción del DANE.

\section{Consideraciones éticas}

El estudio se clasificó como una investigación sin riesgo con base en la Resolución 8430 de 1993 del Ministerio de Salud porque se emplearon técnicas y métodos de investigación documental retrospectivos y no se realizó ninguna intervención o modificación intencionada de las variables biológicas, fisiológicas, sicológicas o sociales de la población en estudio, por lo tanto, no se incurrió en ninguna falta bioética al ser humanoy no se violó el derecho de privacidad ${ }_{(5)}$. La información se obtuvo de bases de datos del SIVIGILA, del RUAF y de los resultados de las encuestas realizadas a nivel nacional. Se tuvieron en cuenta los principios de confidencialidad de la información y responsabilidad. Los resultados de este estudio le aportan al fortalecimiento de la vigilancia de VIH - SIDA y mortalidad por SIDA en Colombia.

\section{Resultados}

\section{Descripción del sistema de vigilancia de VIH - SIDA y Mortalidad por SIDA}

En Colombia la notificación de casos de VIH -SIDA y Mortalidad por SIDA se realiza de manera individual. Si bien durante el año 2006 se contemplan los cambios en diferentes variables según el paso del tiempo, desde el 2017 se inició el proceso de consolidación de bases nacionales con un solo registro por paciente, sin importar el cambio de estadio clínico del mismo.

La notificación de casos inicia a partir de la ocurrencia del evento. La Unidad Primaria Generadora de Datos (UPGD) o unidad informadora (UI) registra el dato con el resultado de laboratorio ya que todo caso de contagio y muerte por VIHSIDA debe ingresar como confirmado por laboratorio, con criterios de confirmación donde los pacientes a partir de los 18 meses de edad no gestantes confirman a través de dos pruebas de inmuno ensayo reactivas (rápida o Elisa).

En el caso de los gestantes, se realiza a través de dos pruebas de inmuno ensayo reactivas (rápida o Elisa), más carga viral que debe tener un valor superior a 5.000 copias; si este valor es inferior, se debe confirmar en este caso con Western Blot . En el caso de menores de 18 meses expuestos a infección por VIH-SIDA desde el nacimiento, primero se hace una prueba de carga viral a las cuatro semanas, si es mayor o igual a 
5.000 copias se realiza inmediatamente segunda carga viral, si esta prueba es mayor o igual a 5.000 copias se confirma el diagnóstico. De lo contrario, se realiza carga viral al mes. Si la primera prueba de carga viral a las cuatro semanas está entre 50 y 5.000 copias, se realiza inmediatamente una segunda carga viral y si la prueba es mayor o igual a 5.000 copias, se realiza otra carga viral para confirmar el diagnóstico; de lo contrario se descarta infección por VIH. Si la prueba carga viral a las cuatro semanas es menor de 50 copias, se repite carga viral a los cuatro meses.

En el caso de los menores de 18 meses de edad con criterios clínicos de VIH, sin conocimiento del estado serológico de la madre, se inicia con una primera prueba presuntiva para VIH, Elisa o prueba rápida, si es reactiva se aplica el algoritmo diagnóstico para niños expuestos al VIH-SIDA desde el nacimiento y si el resultado es no reactivo se descarta infección por VIH. Esta notificación se realiza de manera rutinaria semanal en la ficha del evento para VIH SIDA y mortalidad por SIDA bajo el código 850, la Unidad Notificadora de la UPGD o UI pasa a la Unidad Notificadora Municipal (UNM) (Dirección Local de Salud), desde el municipio, se notificarán semanalmente al departamento o distrito (Dirección o Secretaría Departamental o Distrital de Salud, Unidad notificadora departamental - UND) que notifica con periodicidad semanal al INS. Una vez notificado el caso se inicia con acciones de control correspondientes según lo establecido.

A nivel nacional, la notificación se capta en el programa SIVIGILA. Una vez se obtiene el archivo de Excel con la base completa de casos notificados, se realiza la depuración de base teniendo en cuenta los criterios de confirmación de caso y haciendo especial énfasis en los casos que no cumplen dichos criterios, es decir, que tienen fallas en la calidad del dato, buscando la realización de los respectivos ajustes por UPGD notificadora. También se buscan registros duplicados y repetidos por cambio de estadio clínico, se analizan los casos teniendo en cuenta notificados por primera vez, semana epidemiológica, características del comportamiento en variables de persona, tiempo y lugar, distribución según edad, sexo, área, afiliación al SGSSS, mecanismo de transmisión.

La localización geográfica se evalúa según el lugar de procedencia, esta información es útil para orientar acciones de promoción y prevención que ayuden a disminuir la incidencia de casos. En variables como la prevalencia, se debe realizar el análisis por entidad territorial, por municipios, por grupos de edad y sexo según la procedencia ya que la distribución de los casos según estas variables, permite a las autoridades sanitarias detectar cualquier cambio en la epidemiología de esta enfermedad. Este análisis es realizado por profesionales especializados que pertenecen a la Dirección de Vigilancia y Análisis del Riesgo en Salud Pública (DVARSP) del INS en el equipo de Infecciones de Trasmisión Sexual (ITS); a su vez las bases son almacenadas en un archivo histórico que reposa en el grupo SIVIGILA. El sistema permite la captura, procesamiento y generación de datos de forma automática, para luego analizar de manera detallada el evento, la difusión de la información consolidada y analizada se realiza a través de informes mensuales por periodos epidemiológicos, boletines epidemiológicos semanales, informes semestrales y anuales que se encuentran disponibles en la página web del INS.

Por su parte el MSPS, es quien imparte las directrices nacionales relacionadas con el manejo programático, garantiza el diagnóstico y suministro de medicamentos antirretroviral, de igual manera se encarga del seguimiento programático de los casos a nivel nacional.

\section{Evaluación de atributos cualitativos}

\section{a. Simplicidad}

El sistema de vigilancia nacional en salud pública nacional utiliza la ficha de notificación con cara A donde se registran los datos básicos del paciente (sociodemográficos) y UPGD que notifica y la cara B para datos específicos del evento. Estas fichas, cuentan con un instructivo que permite estandarizar la información que se registra. A pesar de que las definiciones de caso han cambiado por los criterios de confirmación, se comprenden fácilmente por los médicos. Sin embargo, se requiere que los médicos de las unidades notificadores tengan un entrenamiento para diligenciar la ficha e identificar las diferentes variables.

Para la evaluación de la simplicidad se tuvo en cuenta la encuesta aplicada a los operadores del sistema de Vigilancia en salud pública y el programa departamental tanto de Caquetá como de otros departamentos, donde se obtuvo una calificación de bueno con tan solo 38,2 puntos. El $80,9 \%$ de los encuestados consideran que el subsistema tiene definiciones de caso comprensibles y más del 75,0\% considera que el diligenciamiento de la ficha y el instructivo son sencillos. El 51,1\% reportan que el tiempo global dedicado a la vigilancia no es apropiado. Otros consideran que se pueden generar conflictos al utilizar SIVIGILA junto a otro aplicativo. El 70,2\% de los encuestados creen que el sistema de vigilancia genera duplicidad de la información, lo cual marca una debilidad. En la Tabla 2 se especifican los datos: 
Tabla 2. Evaluación de los atributos (Simplicidad), Subsistema de vigilancia epidemiológica de VIH - SIDA y mortalidad por SIDA, Caquetá, 2019.

\begin{tabular}{|c|c|c|c|c|c|}
\hline Simplicidad & 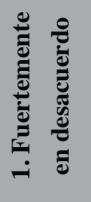 & 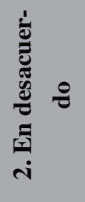 & 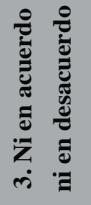 & 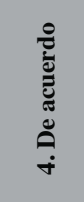 & 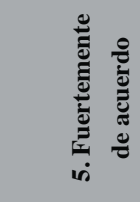 \\
\hline $\begin{array}{l}\text { La definición de caso de VIH, SIDA y mortalidad por SIDA es sencilla y } \\
\text { comprensible }\end{array}$ & 2,1 & 6,4 & 10,6 & 44,7 & 36,2 \\
\hline El diligenciamiento de las variables de la ficha epidemiológica es sencillo & 0,0 & 2,1 & 19,1 & 46,8 & 31,9 \\
\hline $\begin{array}{l}\text { La obtención de los datos que se recolectan para la notificación de un } \\
\text { caso de VIH, SIDA y mortalidad por SIDA es sencillo }\end{array}$ & 0,0 & 4,3 & 21,3 & 48,9 & 25,5 \\
\hline El instructivo de diligenciamiento de la ficha es fácil de comprender & 0,0 & 4,3 & 19,1 & 46,8 & 29,8 \\
\hline $\begin{array}{l}\text { La cantidad de datos a recoger es apropiada para los fines de vigilancia } \\
\text { de la VIH, SIDA y mortalidad por SIDA }\end{array}$ & 2,1 & 6,4 & 12,8 & 57,4 & 21,3 \\
\hline El tiempo global dedicado a la vigilancia es apropiado & 2,1 & 17,0 & 29,8 & 40,4 & 10,6 \\
\hline $\begin{array}{l}\text { Las actividades de vigilancia son sencillas en términos de la trasmisión y } \\
\text { análisis de los datos }\end{array}$ & 0,0 & 4,3 & 19,1 & 53,2 & 23,4 \\
\hline El sistema de vigilancia genera duplicidad de la información. & 4,3 & 6,4 & 19,1 & 44,7 & 25,5 \\
\hline $\begin{array}{l}\text { Existe conflicto por diligenciar datos separados en Sivigila y otros apli- } \\
\text { cativos }\end{array}$ & 8,5 & 12,8 & 29,8 & 25,5 & 23,4 \\
\hline $\begin{array}{l}\text { Todos los datos que se recogen en la vigilancia de la VIH, SIDA y morta- } \\
\text { lidad por SIDA son analizados }\end{array}$ & 4,3 & 8,5 & 27,7 & 42,6 & 17,0 \\
\hline
\end{tabular}

\section{b. Flexibilidad}

El Subsistema de Vigilancia Epidemiológica en VIH - SIDA y Mortalidad por SIDA ha tenido cambios entre el 2006 y 2018 , debido a que se ha modificado la ficha en algunas variables y las guías clínicas de manera anual. Los cambios se han generado en forma concertada con los equipos locales.

En el grupo se identificó que el atributo flexibilidad se cumple con una calificación de bueno con 15,0 puntos. Los actores del sistema creen que los cambios en el sistema han fortalecido la notificación, con el compromiso de más actores. En cuanto a las sugerencias en los cambios, el 44,7\% de los encuestados no están de acuerdo ni en desacuerdo, aunque para más del 50,0\% estos cambios han sido favorables. Los datos se especifican en la Tabla 3: 
Tabla 3. Evaluación de los atributos (Flexibilidad), Subsistema de vigilancia epidemiológica de VIH - SIDA y mortalidad por SIDA, Caquetá, 2019

\begin{tabular}{|c|c|c|c|c|c|}
\hline Flexibilidad & 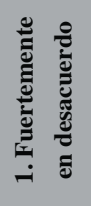 & 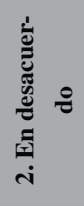 & 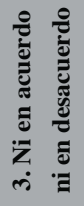 & 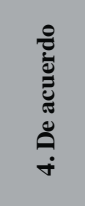 & 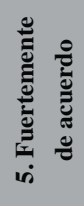 \\
\hline $\begin{array}{l}\text { Identifica los cambios realizados en el proceso de vigilancia de VIH- } \\
\text { SIDA y mortalidad por SIDA desde el año } 2006 \text { al } 2018\end{array}$ & 2,1 & 8,5 & 21,3 & 44,7 & 23,4 \\
\hline El sistema permite incorporar cambios para vigilar un nuevo problema & 0,0 & 8,5 & 25,5 & 44,7 & 21,3 \\
\hline $\begin{array}{l}\text { Los cambios permitieron mejorar el sistema de vigilancia de VIH-SIDA y } \\
\text { mortalidad por SIDA }\end{array}$ & 0,0 & 0,0 & 23,4 & 59,6 & 17,0 \\
\hline $\begin{array}{l}\text { Las sugerencias que usted realiza al sistema de vigilancia son tenidas en } \\
\text { cuenta. }\end{array}$ & 2,1 & 4,3 & 44,7 & 38,3 & 10,6 \\
\hline
\end{tabular}

\section{c. Aceptabilidad}

Más el $80 \%$ de los encuenstados en todos los niveles reconocen la importancia de la vigilancia de VIH - SIDA y mortalidad por SIDA en salud pública. A su vez, el 78,7\% del talento humano está satisfecho con los resultados que le brinda el sistema y creen que se ajusta a las necesidades del territorio. En un 21,3\% de los encuestados no están en acuerdo o desacuerdo en que los participantes de la vigilancia en los diferentes niveles cumplen con las actividades definidas (recolección, envío de datos, análisis de la información y comunicación), por lo tanto, un 10,6\% no identifica alertas. Este atributo obtuvo una calificación de 24,1 puntos lo que lo define como bueno. En la Tabla 4 se especifican los valores:

Tabla 4. Evaluación de los atributos (Aceptabilidad), Subsistema de vigilancia epidemiológica de VIH - SIDA y mortalidad por SIDA, Caquetá, 2019.

\begin{tabular}{|c|c|c|c|c|c|}
\hline Aceptabilidad & 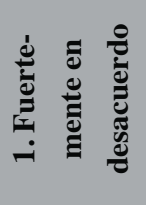 & 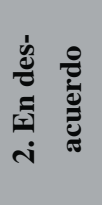 & 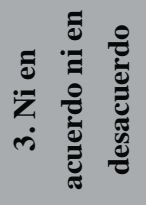 & 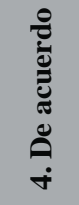 & 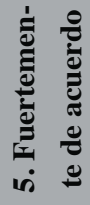 \\
\hline $\begin{array}{l}\text { La vigilancia de VIH, SIDA y mortalidad por SIDA es importante en salud } \\
\text { pública. }\end{array}$ & 2,1 & 0,0 & 0,0 & 12,8 & 85,1 \\
\hline $\begin{array}{l}\text { Los participantes de la vigilancia en los diferentes niveles cumplen con las } \\
\text { actividades definidas (recolección, envío de datos, análisis de la información y } \\
\text { comunicación) }\end{array}$ & 2,1 & 4,3 & 21,3 & 48,9 & 23,4 \\
\hline $\begin{array}{l}\text { Usted identifica las alertas que se pueden generar al realizar la vigilancia de } \\
\text { VIH, SIDA y mortalidad por SIDA }\end{array}$ & 6,4 & 6,4 & 10,6 & 57,4 & 19,1 \\
\hline $\begin{array}{l}\text { El diseño y estructura del sistema de vigilancia se ajusta a las necesidades del } \\
\text { territorio }\end{array}$ & 2,1 & 4,3 & 19,1 & 63,8 & 10,6 \\
\hline
\end{tabular}




\section{d. Oportunidad}

Se evidencia que el análisis de la información del sistema de vigilancia se realiza en cada territorio de manera mensual o trimestral, según lo establezcan los contratos de los actores de este proceso. Por tal periodicidad, más del $65,0 \%$ consideran oportuno el tiempo que transcurre entre notificación de los casos y el análisis de la información. Se considera que no se ha logrado controlar el evento según las acciones recomendadas. Este atributo obtuvo una calificación de regular con 10,4 puntos. Ver tabla 5:

\section{Tabla 5. Evaluación de los atributos (Oportunidad), Subsistema de vigilancia epidemiológica de VIH - SIDA y} Mortalidad por SIDA, Caquetá, 2019.

\begin{tabular}{|c|c|c|c|c|c|}
\hline Oportunidad & 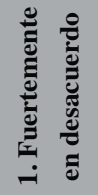 & 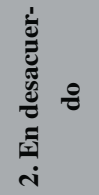 & 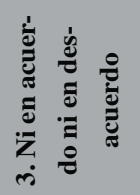 & 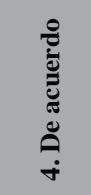 & 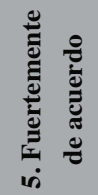 \\
\hline $\begin{array}{l}\text { Considera oportuno el tiempo que transcurre entre notificación } \\
\text { de los casos y el análisis de la información. }\end{array}$ & 2,1 & 8,5 & 23,4 & 51,1 & 14,9 \\
\hline $\begin{array}{l}\text { Se toman medidas para actuar sobre los problemas identificados } \\
\text { y se ejecutan las acciones de vigilancia }\end{array}$ & 2,1 & 10,6 & 21,3 & 53,2 & 12,8 \\
\hline Se ha logrado controlar el evento según las acciones recomendadas & 4,3 & 17,0 & 53,2 & 17,0 & 8,5 \\
\hline
\end{tabular}

\section{e. Representatividad}

Al evaluar la parte cualitativa de este atributo, encontramos que su calificación estuvo por debajo de 8 puntos, lo que indica que es regular. Más del 50,0\% de los entrevistados consideran que los datos obtenidos no representan todos los casos ocurridos en su territorio, aunque este proporciona la información necesaria para caracterizar el comportamiento del VIH -SIDA y mortalidad por SIDA en el territorio. Los datos se especifican en la Tabla 6:

Tabla 6. Evaluación de los atributos (Representatividad), Subsistema de vigilancia epidemiológica de VIH - SIDA y Mortalidad por SIDA, Caquetá, 2019.

\begin{tabular}{|c|c|c|c|c|c|}
\hline Representatividad & 莺 & 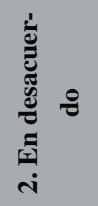 & 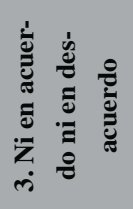 & 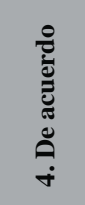 & 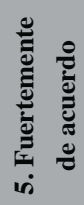 \\
\hline $\begin{array}{l}\text { Considera que los datos obtenidos representan todos los casos ocurridos } \\
\text { en su territorio }\end{array}$ & 6,4 & 31,9 & 21,3 & 29,8 & 10,6 \\
\hline $\begin{array}{l}\text { El sistema de vigilancia proporciona la información necesaria para } \\
\text { caracterizar el comportamiento del VIH - SIDA y mortalidad por SIDA } \\
\text { en Colombia }\end{array}$ & 2,1 & 4,3 & 25,5 & 53,2 & 14,9 \\
\hline
\end{tabular}

\section{f. Utilidad}

Su calificación fue de 19,3 puntos lo que indica que se percibe como buena. Más del 67,0\% de los entrevistados consideran que la vigilancia de VIH -SIDA y mortalidad por SIDA es útil. El 36,2\% de los entrevistados considera que el sistema no permite realizar visita de campo para el control y prevención del VIH -SIDA y mortalidad por SIDA. Ver Tabla 7: 
Tabla 7. Evaluación de los atributos (Utilidad), Subsistema de vigilancia epidemiológica de VIH - SIDA y Mortalidad por SIDA, Caquetá, 2019.

\begin{tabular}{|c|c|c|c|c|c|}
\hline Utilidad & 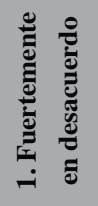 & 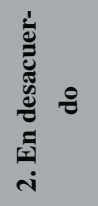 & 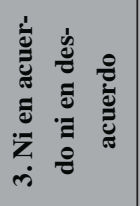 & 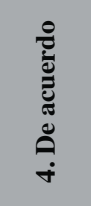 & 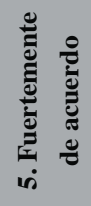 \\
\hline Es útil la vigilancia de VIH -SIDA y mortalidad por SIDA. & 2,1 & 0,0 & 6,4 & 23,4 & 68,1 \\
\hline $\begin{array}{l}\text { Considera que son útiles las acciones que se realizan en la vigilancia del } \\
\text { VIH - SIDA y mortalidad por SIDA. }\end{array}$ & 0,0 & 10,6 & 14,9 & 38,3 & 36,2 \\
\hline $\begin{array}{l}\text { Utiliza la información generada de vigilancia de VIH -SIDA y mortalidad } \\
\text { por SIDA. }\end{array}$ & 2,1 & 6,4 & 4,3 & 44,7 & 42,6 \\
\hline $\begin{array}{l}\text { El sistema permite realizar visita de campo para el control y prevención } \\
\text { del VIH - SIDA y mortalidad por SIDA. }\end{array}$ & 21,3 & 14,9 & 31,9 & 17,0 & 14,9 \\
\hline $\begin{array}{l}\text { Las acciones de vigilancia del VIH - SIDA y mortalidad por SIDA se eva- } \\
\text { lúan mediante el análisis de los indicadores establecidos. }\end{array}$ & 8,5 & 2,1 & 21,3 & 51,1 & 17,0 \\
\hline
\end{tabular}

\section{Evaluación de atributos cuantitativos}

\section{a. Calidad del dato}

Según el flujo de información y las actividades realizadas por los referentes de cada municipio, se asegura la calidad del dato y la veracidad de la información diligenciada en la ficha de notificación. Por niveles del flujo de información el equipo realiza la precrítica de las fichas y la depuración de la información registrada.

Semanalmente, la base departamental es alimentada por las municipales que a su vez recibe los datos de sus UPGD y UI locales. El departamento realiza una revisión de la calidad del dato mediante procesos estandarizados y envía semanalmente esta información al nivel nacional. En esta evaluación se revisó la base de Excel obtenida desde el aplicativo SIVIGILA 2018-2020 del evento 850 de 2014 a 2018, con un total de 623 registros incluyendo registros con ajuste D (error de digitación) y 6 (descartado por laboratorio o unidad de análisis) entre 3 días de nacido y 80 años. Al evaluar los registros no se encontraron registros duplicados (todas las variables iguales), para un resultado de $0 \%$. El porcentaje de registros repetidos (registros con las variables llave iguales), fue de $16,5 \%$ (20 registros repetidos). Los datos se especifican en la Tabla 8 :

Tabla 8. Porcentaje de casos repetidos en la base de datos de VIH - SIDA y Mortalidad por SIDA, Caquetá, 2014-2018

\begin{tabular}{|c|c|c|c|}
\hline Año & Registros analizados & Registros repetidos & Porcentaje \\
\hline 2014 & 97 & 2 & 2,1 \\
\hline 2015 & 134 & 1 & 0,7 \\
\hline 2016 & 141 & 5 & 3,5 \\
\hline 2017 & 103 & 7 & 6,8 \\
\hline 2018 & 148 & 5 & 3,4 \\
\hline
\end{tabular}

Fuente: Sivigila Departamental, 2014-2018.

Respecto a la completitud de variables de importancia para la vigilancia, se encontró que el año con más datos incompletos fue el 2014 con 68,4\% (108 datos ) en las variables y 1,3\% (2 casos) para el 2015; de estas variables "indigente" es el que más dejaron en blanco con el 3,4\%. Ver Tabla 9: 
Tabla 9. Porcentaje de datos faltantes según variables de interés en la vigilancia de VIH - SIDA y mortalidad por SIDA, Caquetá, 2014-2018

\begin{tabular}{|l|r|r|r|r|r|r|}
\hline Variable & \multicolumn{1}{|c|}{$\mathbf{2 0 1 4}$} & \multicolumn{1}{c|}{$\mathbf{2 0 1 5}$} & \multicolumn{1}{c|}{$\mathbf{2 0 1 6}$} & \multicolumn{1}{c|}{$\mathbf{2 0 1 7}$} & \multicolumn{1}{c|}{$\mathbf{2 0 1 8}$} & \multicolumn{1}{c|}{ Total } \\
\hline Indigente & 12,4 & 0,7 & 0,7 & 3,9 & 2,0 & 3,4 \\
\hline Desmovilizados & 11,3 & 0,0 & 0,7 & 1,9 & 2,0 & 2,7 \\
\hline Gestante & 11,3 & 0,7 & 0,7 & 3,9 & 0,0 & 2,7 \\
\hline Víctima de violencia & 11,3 & 0,0 & 0,7 & 1,0 & 2,0 & 2,6 \\
\hline Psiquiátrico & 10,3 & 0,0 & 0,7 & 1,0 & 2,0 & 2,4 \\
\hline Migrante & 11,3 & 0,0 & 0,7 & 1,0 & 1,4 & 2,4 \\
\hline Discapacitados & 11,3 & 0,0 & 0,7 & 1,0 & 1,4 & 2,4 \\
\hline Desplazado & 11,3 & 0,0 & 0,7 & 0,0 & 1,4 & 2,2 \\
\hline Población ICBF & 11,3 & 0,0 & 0,7 & 0,0 & 1,4 & 2,2 \\
\hline Privada de la libertad & 4,1 & 0,0 & 0,0 & 1,0 & 0,7 & 1,0 \\
\hline Madres Comunitaria & 4,1 & 0,0 & 0,7 & 0,0 & 0,7 & 1,0 \\
\hline Otros & 1,0 & 0,0 & 0,7 & 0,0 & 0,0 & 0,3 \\
\hline Total & 68,4 & 1,3 & 7,0 & 9,5 & 13,9 & 100,0 \\
\hline
\end{tabular}

Fuente: Sivigila Departamental, 2014-2018.

\section{b. Sensibilidad}

Por el método captura y recaptura el número total de casos con condición final muerto para el evento de VIH - SIDA y mortalidad por SIDA en el 2014-2018 es de 124 casos con un IC95\%, la sensibilidad del SIVIGILA para captar casos con condición final muerto es del 47,8\% (55), mientras el RUAF detecta el 92,7\% (115) de los casos. Ver Tabla 10:

Tabla 10. Casos de VIH - SIDA y mortalidad por SIDA notificados con condición final muerto en Sivigila y RUAF, Caquetá, 2014-2018

\begin{tabular}{|c|c|c|c|c|}
\hline & & \multicolumn{3}{|c|}{ Ruaf } \\
\hline & & Reportados & No reportados & Total \\
\hline \multirow{3}{*}{ Sivigila } & Notificados & 55 & & 64 \\
\hline & No notificados & 60 & & 60 \\
\hline & Total & 115 & & 124 \\
\hline
\end{tabular}

Fuente: Sivigila Departamental, 2014-2018

\section{c. Valor Predictivo Positivo}

El valor predictivo positivo del SIVIGILA para identificar verdaderas muertes por VIH - SIDA y mortalidad por SIDA en Caquetá del 2014-2018 de SIVIGILA y RUAF es de 85,9\% (55/55+9).

\section{d. Especificidad:}

El SIVIGILA arrojó una especificidad del 10,0\% (1/9+1) para captar correctamente casos con condición final muertos que se encuentran registrados en RUAF como condición en alguna de las causas de muerte VIH - SIDA.

\section{e. Oportunidad:}

La oportunidad en la notificación fue de 165,8 días en promedio, con un valor mínimo de cero días y máximo de 4.439 días. 


\section{f. Representatividad:}

Según el sexo, la prevalencia es mayor para el masculino durante todos los años. En el departamento, la prevalencia más alta entre los cinco años de estudio fue en el 2018 con 31,8 casos por 100.000 habitantes hombres del departamento. Ver tabla 11:

Tabla 11. Prevalencia de VIH-SIDA y mortalidad por SIDA por sexo, Caquetá, 2014-2018.

\begin{tabular}{|c|c|c|c|c|c|c|c|c|c|c|}
\hline \multirow{2}{*}{ SEXO } & \multicolumn{2}{|c|}{$\mathbf{2 0 1 4}$} & \multicolumn{2}{c|}{$\mathbf{2 0 1 5}$} & \multicolumn{2}{|c|}{$\mathbf{2 0 1 6}$} & \multicolumn{3}{|c|}{$\mathbf{2 0 1 7}$} & \multicolumn{2}{|c|}{$\mathbf{2 0 1 8}$} \\
\cline { 2 - 12 } & Casos & Prevalencia & Casos & Prevalencia & Casos & Prevalencia & Casos & Prevalencia & Casos & Prevalencia \\
\hline Femenino & 28 & 11,9 & 34 & 14,3 & 29 & 12,0 & 17 & 6,9 & 35 & 14,1 \\
\hline Masculino & 35 & 14,8 & 48 & 20,0 & 53 & 21,9 & 50 & 20,4 & 79 & 31,8 \\
\hline
\end{tabular}

Fuente: Sivigila Departamental, 2014-2018.

Según el grupo etario, al revisar el comportamiento de VIH - SIDA y mortalidad por SIDA según clasificación del riesgo por año (semaforización), en número de casos y prevalencia según cuartiles para el periodo 2014 - 2018 en los casos notificados al SIVIGILA, se observa en todos los años prevalencias altas por encima del cuartil 0,75 en por lo menos 4 grupos de edad; excepto para el 2017 donde solo se observa prevalencia alta para el grupo de 30-34 años. Para el 2015, se observa mayor prevalencia para los grupos de 20 a 29 años y 40 a 49 años; en el 2016 en los grupos de 20 a 29 años y de 35 a 44 años; en el 2018 de 20 a 34 años. Ver tabla 12.

Tabla 12. Prevalencia de VIH-SIDA y mortalidad por SIDA por grupo de edad, Caquetá, 2014-2018.

\begin{tabular}{|c|c|c|c|c|c|c|c|c|c|c|}
\hline \multirow{2}{*}{$\begin{array}{c}\text { Grupo de } \\
\text { edad }\end{array}$} & \multicolumn{2}{|r|}{2014} & \multicolumn{2}{|r|}{2015} & \multicolumn{2}{|r|}{2016} & \multicolumn{2}{|r|}{2017} & \multicolumn{2}{|r|}{2018} \\
\hline & Casos & Prevalencia & Casos & Prevalencia & Casos & Prevalencia & Casos & Prevalencia & Casos & Prevalencia \\
\hline$<1$ año & 0 & 0,0 & 0 & 0,0 & 2 & 17,9 & 0 & 0,0 & 0 & 0,0 \\
\hline $5-9$ & 2 & 3,8 & 0 & 0,0 & 0 & 0,0 & 0 & 0,0 & 2 & 3,8 \\
\hline $10-14$ & 0 & 0,0 & 0 & 0,0 & 0 & 0,0 & 0 & 0,0 & 1 & 1,9 \\
\hline $20-24$ & 4 & 9,0 & 16 & 35,4 & 19 & 41,6 & 13 & 28,2 & 18 & 38,6 \\
\hline $25-29$ & 6 & 15,4 & 17 & 42,4 & 16 & 39,1 & 12 & 28,8 & 18 & 42,7 \\
\hline $30-34$ & 15 & 48,3 & 9 & 28,2 & 7 & 21,2 & 16 & 46,7 & 17 & 47,6 \\
\hline 35 - 39 & 11 & 40,2 & 6 & 21,5 & 11 & 38,9 & 9 & 31,5 & 9 & 31,0 \\
\hline 55 - 59 & 1 & 6,4 & 3 & 18,4 & 1 & 5,9 & 3 & 17,1 & 3 & 16,4 \\
\hline $60-64$ & 5 & 40,2 & 4 & 31,1 & 1 & 7,5 & 0 & 0,0 & 4 & 28,1 \\
\hline$>65$ años & 0 & 0,0 & 1 & 3,8 & 1 & 3,7 & 1 & 3,6 & 2 & 6,9 \\
\hline
\end{tabular}

Fuente: Sivigila Departamental, 2014-2018.

A nivel municipal, se encontró que los municipios de Albania y Florencia presentan las prevalencias más altas en 4 de los 5 años del estudio, con un promedio de 24,9 casos por 100.000 habitantes para Albania y 33,1 casos para Florencia. Ver tabla 13. 
Tabla 13. Prevalencia de VIH-SIDA y mortalidad por SIDA por municipio de residencia, Caquetá, $2014-2018$.

\begin{tabular}{|c|c|c|c|c|c|c|c|c|c|c|}
\hline \multirow[b]{2}{*}{ Municipio } & \multicolumn{2}{|c|}{2014} & \multicolumn{2}{|c|}{2015} & \multicolumn{2}{|c|}{2016} & \multicolumn{2}{|c|}{2017} & \multicolumn{2}{|c|}{2018} \\
\hline & 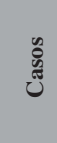 & 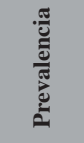 & 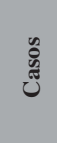 & 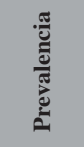 & 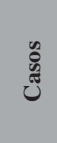 & 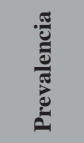 & 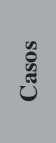 & 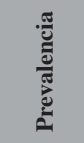 & 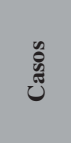 & 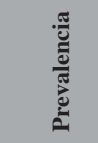 \\
\hline Albania & 1 & 15,6 & 0 & 0,0 & 3 & 46,6 & 3 & 46,6 & 1 & 15,5 \\
\hline Belén de los Andaquíes & 0 & 0,0 & 0 & 0,0 & 0 & 0,0 & 2 & 17,1 & 1 & 8,5 \\
\hline Cartagena del Chairá & 1 & 3,0 & 7 & 21,0 & 9 & 26,5 & 1 & 2,9 & 4 & 11,4 \\
\hline Curillo & 0 & 0,0 & 2 & 17,1 & 1 & 8,5 & 1 & 8,5 & 0 & 0,0 \\
\hline El Doncello & 2 & 9,1 & 2 & 9,0 & 2 & 9,0 & 3 & 13,5 & 3 & 13,5 \\
\hline El Paujil & 1 & 5,0 & 0 & 0,0 & 1 & 4,9 & 0 & 0,0 & 3 & 14,2 \\
\hline Florencia & 50 & 29,5 & 60 & 34,8 & 54 & 30,8 & 46 & 25,8 & 81 & 44,6 \\
\hline Montañita & 0 & 0,0 & 3 & 12,7 & 1 & 4,2 & 2 & 8,3 & 3 & 12,4 \\
\hline Milán & 0 & 0,0 & 0 & 0,0 & 0 & 0,0 & 2 & 16,9 & 0 & 0,0 \\
\hline Morelia & 0 & 0,0 & 0 & 0,0 & 0 & 0,0 & 0 & 0,0 & 0 & 0,0 \\
\hline Puerto Rico & 1 & 3,0 & 2 & 6,0 & 3 & 9,0 & 0 & 0,0 & 2 & 5,9 \\
\hline San José de Fragua & 1 & 6,7 & 2 & 13,4 & 4 & 26,6 & 1 & 6,6 & 3 & 19,7 \\
\hline San Vicente del Caguán & 2 & 3,0 & 2 & 2,9 & 3 & 4,3 & 2 & 2,8 & 6 & 8,4 \\
\hline Solano & 0 & 0,0 & 0 & 0,0 & 0 & 0,0 & 0 & 0,0 & 3 & 12,0 \\
\hline Solita & 1 & 10,9 & 1 & 10,9 & 0 & 0,0 & 1 & 10,9 & 1 & 10,9 \\
\hline Valparaíso & 1 & 8,6 & 0 & 0,0 & 0 & 0,0 & 0 & 0,0 & 1 & 8,5 \\
\hline Desconocido Caquetá & 2 & 0,4 & 1 & 0,2 & 1 & 0,2 & 3 & 0,6 & 2 & 0,4 \\
\hline Caquetá & 63 & 13,4 & 82 & 17,2 & 82 & 16,9 & 67 & 13,7 & 114 & 23,0 \\
\hline
\end{tabular}

Fuente: Sivigila Departamental, 2014-2018.

\section{Discusión}

El Sistema de Vigilancia de VIH / SIDA y Mortalidad por SIDA capta y permite conocer el comportamiento de la enfermedad produciendo información esencial para la toma de decisiones. En general, el sistema cumple con las cualidades y ventajas que debe tener un sistema de vigilancia.

Con base en la encuesta aplicada, los participantes consideran que el sistema de vigilancia en salud pública para VIH - SIDA y Mortalidad por SIDA es simple en cuanto a su diligenciamiento de fichas epidemiológicas, proceso de notificación, estructura y operación del sistema de vigilancia. Igualmente, lo consideran flexible y aceptable, reconociendo como positivo solo dejar un registro sin importar el estadio clínico, mejorando así el proceso de notificación y análisis. Sin embargo, la representatividad y oportunidad se consideran aceptables.

La calidad de los datos recolectados frecuentemente mide la eficacia de un sistema de vigilancia, siendo este un atributo de los más importantes en el momento de realizar análisis. En Colombia, el SIVIGILA efectúa automáticamente un proceso de depuración de registros duplicados, es decir, elimina los registros idénticos en todas sus variables, es por ello por lo que no se encontraron registros duplicados para este evento en el departamento de Caquetá ${ }_{(6)}$. Se encontró un $3,1 \%$ de registros repetidos, para el porcentaje de variables en blanco o faltantes, no se obtuvo porcentajes superiores al 15,0\%, reflejando un buen diligenciamiento de las fichas de notificación individual y complementaria por los operadores del sistema, siendo este insumo importante para el análisis de la información e indicadores propios del evento.

A pesar de que el atributo de utilidad fue bueno, en algunos municipios la información obtenida es impedida para análisis, generación de informes, priorización de necesidades en salud pública, boletines, uso académico en grupos de investigación, entre otras, ${ }_{(7)}$ 
debido al desconocimiento de la información que registra el SIVIGILA por algunos usuarios del sistema de vigilancia.

En relación con la oportunidad del sistema de vigilancia, no se cumple con la oportunidad en la notificación de casos por el evento. Para este estudio la diferencia entre la fecha de consulta y la fecha de notificación fue en promedio de 165 días, superior a lo establecido por el lineamiento nacional, evidenciándose debilidades en el proceso de notificación en general, probablemente debido a que este proceso está sujeto a trámites administrativos de cada entidad territorial, rotación del personal y dificultades de acceso a internet para realizar este proceso ${ }_{(8)}$.

Dentro de los atributos cuantitativos se encuentra la sensibilidad que permite conocer la capacidad del sistema para identificar casos con condición final muerto de VIH - SIDA en la población Caqueteña. Para este estudio, el SIVIGILA captó casi la mitad de los casos con condición final muerte mientras que en RUAF fue cerca del 95,0\%. La diferencia entre los datos probablemente se explica debido a que el SIVIGILA no es una fuente para capturar casos con condición final muerto, la herramienta de registro de casos fallecidos en el país es RUAF-ND, en el SIVIGILA la notificación y ajuste de los casos no se hace con la exactitud puesto que el Sivigila solo permite ingresar una causa mediante código CIE10 y el personal de salud que tiene a cargo el diligenciamiento de la ficha de notificación y digitación en el aplicativo desconoce la codificación o no la emplea adecuadamente, lo que lleva a debilidades en la correspondencia de las dos fuentes ${ }_{(9)}$.

El SIVIGILA cuenta con un valor predictivo positivo alto (superior al 85\%) para captar casos con condición final muerte registrados por RUAF y SIVIGILA, con una alta probabilidad de que un caso notificado por el sistema con condición final muerto realmente haya fallecido por una de las complicaciones del SIDA.

Al realizar el análisis de representatividad entre las dos fuentes oficiales con las que cuenta el territorio nacional, se encuentra que los datos registrados en el SIVIGILA no muestran diferencias en variables de edad y sexo con las reportadas por el Programa nacional, siendo esta una fuente confiable y que da cuenta del total de casos tratados en el país. No se calcularon diferencias entre otras variables demográficas y sociales, debido a que el programa no maneja datos nominales, sino agrupados, diferencia con la información registrada por el SIVIGILA, que si da cuenta de estas variables en tiempo real ${ }_{(10,11)}$.

\section{Conclusiones}

De acuerdo con los resultados de este estudio, la estructura del Sistema de Vigilancia epidemiológica (SIVIGILA) de VIH SIDA y mortalidad por SIDA en Caquetá cuenta con procesos organizados que permiten captar, notificar, consolidar y analizar la información de una forma adecuada, para orientar la toma de decisiones a nivel local y departamental.

Los atributos cualitativos -simplicidad, flexibilidad y aceptabilidad- tuvieron en general un buen desempeño con respecto a las escalas establecidas. Por su parte, los atributos de oportunidad y representatividad se clasificaron como regulares. En general, el sistema de vigilancia se considera simple y permite recolectar datos de una forma sencilla por medio de las fichas epidemiológicas.

$\mathrm{Al}$ analizar el comportamiento que tiene el sistema de vigilancia de VIH - SIDA y mortalidad por SIDA en Caquetá para captar casos con condición final muerto, se pudo identificar que el SIVIGILA cuenta con una sensibilidad menor que el RUAF, ya que esta última fuente es la oficial en el país para capturar esta información, con un valor predictivo positivo sobre el $85 \%$.

Al comparar el número de casos del sistema de vigilancia con otros reportes de mortalidad, se evidencia que RUAF identifica un mayor número de casos con condición final muerte, por lo cual, se puede considerar la fuente más representativa para obtener información de mortalidad en el departamento de Caquetá.

\section{Referencia bibliográficas}

1.Instituto Nacional de Salud. Protocolo VIH-SIDA y mortalidad por SIDA. [Internet ]2018 [consultado 2019 Abr 13]. Disponible en: https://www.ins.gov.co/buscador-eventos/ Lineamientos/PRO\%20VIH\%20sida _pdf.

2. Organización Mundial de la Salud. Infecciones de transmisión sexual. [Internet ]2019. Disponible en: https:// www.who.int/es/news-room/fact-sheets/detail/sexuallytransmitted-infections-(stis). [consultado 2019 Abr 13]

3. Instituto Nacional de Salud. Informe de evento VIH-SIDA y mortalidad por SIDA. [consultado 2019 Abr 13] Disponible en: https://www.ins.gov.co/buscador-eventos/Informesdeevento/ VIH-SIDA\%202017.pdf.

4. Center for Disease Control and Prevention. Updated Guidelines for Evaluating Public Health Surveillance Systems. Recommendations from the Guidelines Working Group. MMWR Recomm reports. [Internet ]2001[consultado 
2019 Abr 13]; 50:p.1-35. Disponible en: http://www.cdc.gov/ mmwr/preview/mmwrhtml/rr5013a1.htm.

5.República de Colombia. Ministerio de Salud. Resolución N ${ }^{\circ}$ 008430 De 1993. 4 De octubre De 1993. Disponible en: http:// www.cdc.gov/mmwr/preview/mmwrhtml/rr5013a1.htm.

6.Instituto Nacional de Salud. Manual del Usuario Sistema Aplicativo Sivigila. [Internet ]2019[consultado 2019 Abr 13]. Disponible en: http://www.ins.gov.co/lineas-deaccion/Subdireccion-Vigilancia/sivigila/Documentos\%20 SIVIGILA/_Manual\%20Sivigila\%202019.pdf.

7.Arias Peña, Díaz A, Andrade I. Evaluación de los atributos del sistema de vigilancia en salud pública en el departamento del Huila durante el 2008. RFS. 2010 abril; 2(1).Disponible en: https://www.journalusco.edu.co/index.php/rfs/article/view/29

8.Organización Panamericana de la Salud. Módulos de principios de epidemiología para el control de enfermedades (MOPECE). Módulo. Washington D.C: Organización Panamericana de la Salud; 2011. Report No.: 9275324077.

9.Leal-Mateos Manrique, Salazar Solís Ricardo, Murillo Calderón Adriana. Evaluación del sistema de vigilancia epidemiológica para la enfermedad diarreica aguda en el área rectora de salud de Alajuela 2. Rev. costarric. salud pública [Internet] 2005 [consultado 2019 Abr 13]. Disponible en: http:// www.scielo.sa.cr/ scielo.php?script=sci_arttext\&pid=S1409$14292005000100005 \& \operatorname{lng}$

10. Arranz N, Pelayo T, Viloria L, San Emeterio G, et al. Evaluación del sistema de vigilancia epidemiológica de la tuberculosis pulmonar en Cantabria durante el periodo 20022004. Boletín. España: Instituto de Salud Carlos III, Centro Nacional de epidemiología; 2006. Report No.: p.1135 - 6286.

11.Batista Moliner Ricardo, González Ochoa Edilberto. Evaluación de la vigilancia en la atención primaria de salud: una propuesta metodológica. Rev. Cubana Med Trop. 2000; 52(1):p. 55-57. 